\title{
Adjusted recommended values of the fundamental physical constants
}

\section{An overview of the data and results of the CODATA-2006 adjustment}

\author{
S.G. Karshenboim ${ }^{\mathrm{a}}$ \\ D.I. Mendeleev Institute for Metrology (VNIIM), St. Petersburg 190005, Russia \\ and Max-Planck-Institut für Quantenoptik, 85748 Garching, Germany
}

\begin{abstract}
A brief overview of the CODATA-2006 adjustment (P.J. Mohr, B.N. Taylor, and D.B. Newell, CODATA Recommended values of the fundamental physical constants: 2006, Rev. Mod. Phys. 80, (2008) 633) is presented. The attention is focussed on the most important data that appeared since the previous adjustment in 2002, and on changes in the recommended values of 2006 in comparison with the results of 2002 . We consider a structure of the input data and their correlations and analyze the results and the data group by group. We also discuss consequences of the adjustment.
\end{abstract}

\section{Introduction}

Fundamental constants play an important role in physics and metrology. They appear in different contexts and different authors understand fundamental constants quite differently. In this paper I will consider 'practical fundamental constants', accurate determination of which is considered in [1]. Such constants may not always have 'truly' fundamental nature, but they are in one or other way related to precision measurements.

In physics, once we need to issue a theoretical prediction we can easily write down related expressions, but that is not enough. To predict numbers, we need some numbers to begin with. Any theory, even ab initio theory, is usually not a construction, which starts from nothing and produces quantitative predictions. Usually, theory is a kind of constraint, which explains relations between results of different experiments. Once we use data of some measurements as input parameters, we can predict the results of other measurements. The most universal input parameters are recognized as fundamental constants of physics.

Even in pure experimental physics we also need fundamental constants. That is because we need reference values for measurements. It is helpful if such reference quantities are somewhat universal. That makes them be useful as units. The most universal of such quantities are not necessary of fundamental nature. The metric system started from quite universal reference values, such as the size of Earth, density of water and the astronomical year. However, such non-fundamental quantities can be applied only with a limited accuracy and beyond a certain limit their non-universality and non-constancy can be observed. Part of universal reference values is stable and well defined in natural terms. Those are usually fundamental constants.

In metrology we need fundamental constants for reasons similar to experimental physics. However, we prefer there to keep certain hierarchy in reference values and only very few constants can serve as units, while the rest are conversion factors.

The status of determination of numerical values of the most important 'practical' fundamental constants is reviewed by the CODATA task group on fundamental constants which regularly performs 'adjustment' of fundamental constants and publishes their recommended values.

\footnotetext{
a e-mail: savely.karshenboim@mpq.mpg.de
} 
The task group was created in 1969 and published since that several adjustments: 1973 [2], 1986 [3], 1998 [4], 2002 [5], and 2006 [1], with the last three adjustments being labeled by the deadline of the collection of the input data. The task group [6] works under auspice of the Committee on Data for Science and Technology (CODATA) of the International Council for Science (ICSU).

\section{What is the adjustment?}

The adjustment occupies a very specific place in physics and metrology. Usually, we deal with a set of similar data. For instance, we have values of the same quantity, say, a frequency $f(P)$ which depends on parameters such as laser power $P$. We perform a certain fit and find a zero-power frequency $f(0)$ and sensitivity parameter $(\partial f / \partial P)_{P=0}$. All the data points have approximately the same accuracy (at least of the same order) and are obtained by essentially the same methods.

In the case of fundamental constants, the situation is very different. Because of their universality they enter equations from very different branches of physics. The results are of very different accuracy and strongly correlated. For instance, we can measure $e^{2} / h, h, e / h, e N_{A}, h N_{A}$, and $e$ etc. At the first place, we should be sure that the data are consistent. The consistency of data means a consistency of reference devices (if any where applied), of methods, and eventually of the results.

From the metrological point of view, testing the reference devices, i.e., the standards, is a crucial metrological problem and the adjustment allows to perform a certain cross-check of electric standards. By checking the consistency of the methods we test basic physical laws and our ability for their successful practical realization for present and future standards. Checking the consistency of the data we check whether the realization of some units is consistent with the rest of the precision physics.

Since we deal with various combinations of the constants, knowledge of the recommended values of a 'minimal independent set' of the constants is not sufficient. For instance, we cannot find $e / h$ from values of $e$ and $h$. We need to know their correlation. For this reason the final resulting tables of the adjustment include many fundamental constants far beyond the 'minimal set'. The CODATA recommended values tables consist of various combinations of constants from such a minimal set.

As we mentioned, the very first and the most important stage is a critical examination of the data. We have to test reliability of the data. In principle, the CODATA evaluation, as any based on the least-square method, essentially relies on the most accurate data of each kind. From the mathematical point of view, we could dramatically reduce the number of experiments under consideration and neglect most of correlations. However, at the stage of a study of consistency and reliability we should examine a quite broad range of data.

Less accurate data, which perhaps would marginally contribute to the final results, are nevertheless studied carefully. Once all the data are consistent, the less accurate data are of marginal importance. However, if we have any doubt in reliability and consistency of the all related data, that is the less accurate data which determine how we should proceed.

Another important issue is strong involvement of theory. Some theoretical expressions keep their naive form up to very high accuracy, like those for the von Klitzing and Josephson constants

$$
\begin{aligned}
R_{\mathrm{K}} & =\frac{h}{e^{2}} \\
K_{\mathrm{J}} & =\frac{2 e}{h},
\end{aligned}
$$

while the others like the hydrogen frequencies needed to determine the Rydberg constant $R_{\infty}$ are very complicated. There is a number of quantities the accurate determination of which strongly depends on the reliability of quite advanced theory. 


\section{Structure of the data}

As we already mentioned, different data are known with a different accuracy and that makes a kind of 'group structure' of the adjustment. If the data are consistent, we can perform the adjustment group by group and the difference between results of this simplified procedure and of the overall adjustment of the data, which was in fact performed in [1], is marginal. The present data set is consistent in general and its group-by-group evaluation is possible.

The group data of the present and few past adjustments suggests (see [7-9] for more explanations)

- the most accurate data form a group of auxiliary data which are to be evaluated prior to the main procedure;

- the main procedure deals with two groups; the most accurate of them is formed by the data related to the fine structure constant $\alpha$;

- the other group of the main adjustment combines the data related to the determination of the elementary charge $e$ and the Planck constant $h$;

- there is also a certain number of constants, as the Boltzmann constant $k$ and the gravitation constant $G$, which are tabulated in the recommended values, but they are independent and are not really involved into the adjustment procedure;

- a certain number of constants are closely correlated with the adjusted values, as, e.g., the electron mass $m_{e}$ (in kilograms); in fact they do not participate in the adjustment of the input data and in one or other way they are derived from the result of the adjustment.

Technically, the adjustment [1] was performed in the following way: at first, the input data were carefully analyzed group by group; next, the input data set was modified based on this analysis (in such a modification some data might be omitted, while for some others a certain expansion of the uncertainty might be introduced); and, eventually, an overall adjustment of the data was performed producing the recommended values of fundamental constants.

We note, however, that the group structure is determined not by the way the data are treated, but by their accuracy, which determines, through various statistical weight factors, the directions in which the information propagates. E.g., we can run an overall least square adjustment and check consequences of removing some pieces of data. In particular, we can easily see that deleting various input data from the auxiliary group would strongly affect contributions of the partial data from the $\alpha$ group, while deleting any $h$ related and independent data would not change the result for the fine structure constant. Thus, we note that the experimental information propagates in an obvious direction: from more accurate data groups to less accurate ones.

Below we shortly review all kinds of the data.

\section{Auxiliary data}

Before discussing the most accurate data of the adjustment, i.e. the auxiliary data, let us remind that a few constants are known exactly because of the definitions of the SI system [10] or other units (like the unified atomic mass unit). Those are collected in the upper part of Table 1.

The auxiliary constants include various atomic masses in the unified atomic mass units, mass ratios and the Rydberg constant

$$
R_{\infty}=\frac{\alpha^{2} m_{e} c}{2 h}
$$

The latter is mostly determined by the adjustment [1] of data from many transitions in atomic hydrogen and deuterium. There are also some specific rather inaccurate data which are needed for small additive corrections, like parameters of weak interactions, which marginally affect some QED calculations relevant for the evaluation in [1].

In general, there has been no essential changes in this sector of adjustment comparing the former [5] and the recent [1] adjustments. 
Table 1. Exactly known constants (upper part) and some auxiliary constants in the adjustment [1]. Here, $u_{r}$ stands for the fractional uncertainty.

\begin{tabular}{lccc}
\hline Quantity & Symbol & Value & $u_{r}$ \\
\hline speed of light in vacuum & $c$ & $299792458 \mathrm{~m} \mathrm{~s}^{-1}$ & exact \\
magnetic constant of vacuum & $\mu_{0}$ & $4 \pi \times 10^{-7} \mathrm{~N} \mathrm{~A}^{-2}$ & exact \\
atomic mass of ${ }^{12} \mathrm{C}$ & $m\left({ }^{12} \mathrm{C}\right)$ & $12 \mathrm{u}$ & exact \\
\hline Rydberg constant & $R_{\infty}$ & $10973731.568527(73) \mathrm{m}^{-1}$ & {$\left[6.6 \times 10^{-12}\right]$} \\
proton-electron mass ratio & $m_{p} / m_{e}$ & $1836.15267247(80)$ & {$\left[4.3 \times 10^{-10}\right]$} \\
proton mass & $m_{p}$ & $1.00727646677(10) \mathrm{u}$ & {$\left[1.0 \times 10^{-10}\right]$} \\
\hline
\end{tabular}

\section{The $\alpha$ connection}

The group related to the fine structure constant involves many data. The most important data points are presented in Fig. 1. There are sixteen different points obtained within six completely different approaches. Two of them involve electric standards, one needs material metrology and three are based on quantum mechanics and quantum electrodynamics and essentially do not involve standards. Many data points assume not a single measurement, but a chain of experiments.

There has been important progress in the field between two adjustments, which produced essential change in the $\alpha$ accuracy.

In both evaluations $[1,5]$ the dominant source is due to a study of the anomalous magnetic moment of the electron. In the case of [5] that was a result based on a single-group measurement and a single-group theory. Theory has improved $[11]^{1}$ (the theoretical uncertainty in $\alpha$ changed from $u_{r}=9.9 \times 10^{-10}$ in the 2002 adjustment to [5] $u_{r}=2.4 \times 10^{-10}$ in the recent adjustment [1]), but it is still based on the activity of a single group ( $\mathrm{T}$. Kinoshita and collaborators). The experimental accuracy was also improved, but by another group. The more recent measurement from Harvard University [12] is essentially more accurate than the former Washington University result (see Fig. 1). Eventually, the best $\alpha\left(a_{e}\right)$ value in 2006 [13] is about five times more accurate than the CODATA 2002 value.

Because of importance of the fine structure constant determination it is desirable to reach an accurate value by a different method. The second accurate method is based on recoil spectroscopy. In the former adjustment [5] only one result was available (a caesium value from Stanford). The CODATA 2006 adjustment [1] deals also with a Rb result from Paris [14]. The most accurate $\alpha$ results are presented in the bottom plot in Fig. 1.

Crucial $\alpha$ related recommended values [1] are presented in Table 2, which includes

$$
\alpha=\frac{e^{2}}{4 \pi \epsilon_{0} h c}
$$

the von Klitzing constant (1), Compton wavelength of electron, and the molar Planck constant.

Since we know the Rydberg constant (2) with a high accuracy, we can easily interpret any result for $\alpha$ in terms of $h /\left(m_{e} c\right)$ and vice versa. Because of the high accuracy achieved for various mass ratios we can easily go further from $h /\left(m_{e} c\right)$ to the value of $h /(m c)$ for a broad range of atoms.

Appearance of the molar Planck constant can be easily understood once we recall that there are two mass-related microscopic units in which we can accurately express various atomic masses. One of them is the frequency unit (i.e. we deal with $m c^{2} / h$ instead of $m$ ) while the other is a unified atomic mass unit. The conversion factor between them is the molar Planck constant and it is limited by the accuracy of measurements in the frequency units.

${ }^{1}$ We present here only references to the most recent data used in [1]. For more references see [5]. 

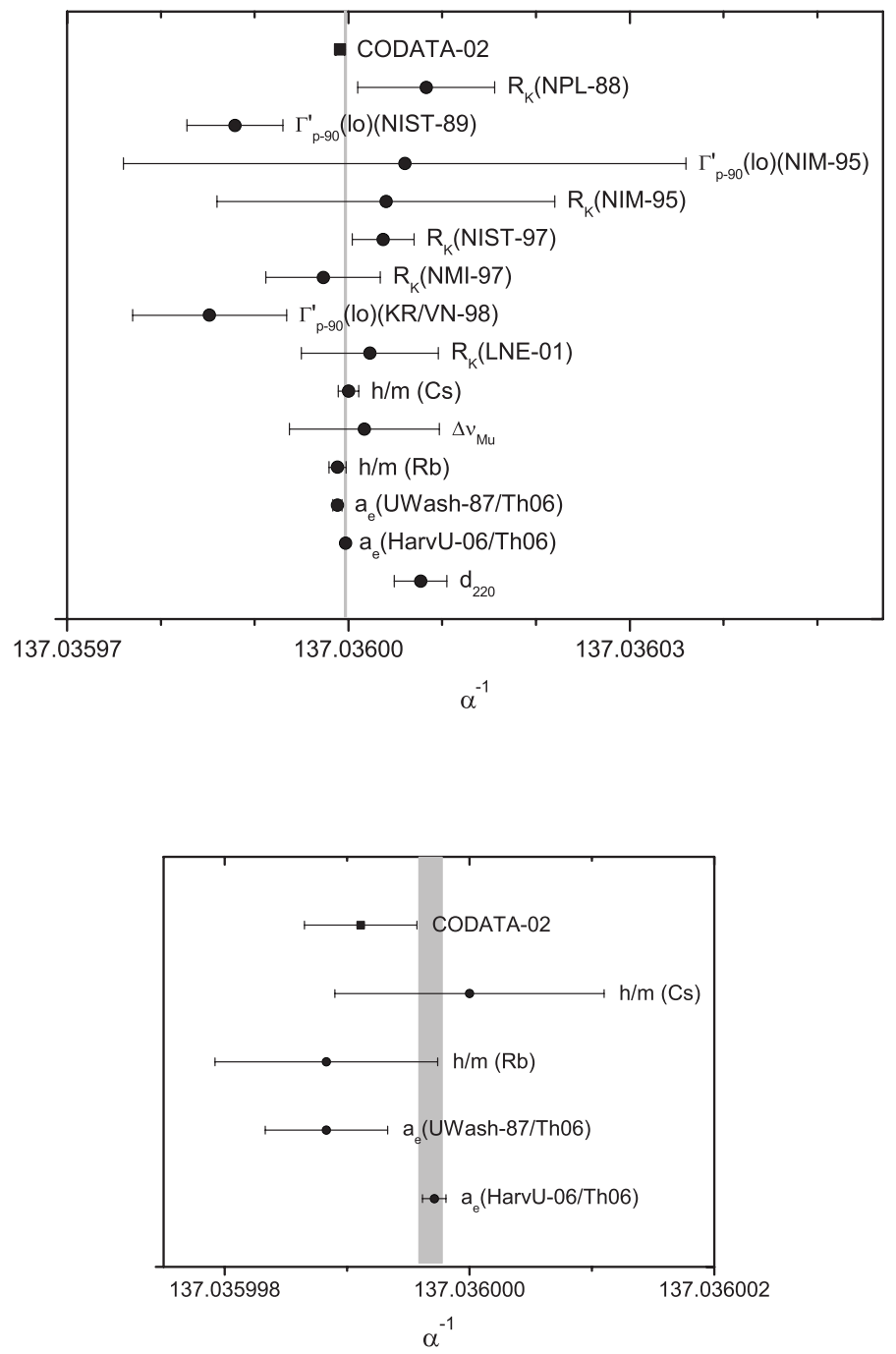

Fig. 1. Determination of the fine structure constant $\alpha$ in the CODATA-2006 adjustment [1]. The labels are similar to those in [1]. The vertical band stands for the adjusted CODATA-2006 value. The most accurate data from $a_{e}$ and recoil spectroscopy are presented in the bottom plot with a magnified scale.

Table 2. Some $\alpha$ related data [1]. Here, $u_{r}$ stands for the fractional uncertainty.

\begin{tabular}{lccc}
\hline Quantity & Symbol & Value & $u_{r}$ \\
\hline inverse fine structure constant & $\alpha^{-1}$ & $137.03599968(9)$ & {$\left[6.8 \times 10^{-10}\right]$} \\
molar Planck constant & $h \cdot N_{A}$ & $3.9903126821(57) \times 10^{-10} \mathrm{~J} \mathrm{~s} \mathrm{~mol}^{-1}$ & {$\left[1.4 \times 10^{-9}\right]$} \\
electron Compton wavelength & $h /\left(m_{e} c\right)$ & $2.4263102175(33) \times 10^{-12} \mathrm{~m}$ & {$\left[1.4 \times 10^{-9}\right]$} \\
von Klitzing constant & $R_{\mathrm{K}}$ & $25812.807557(18) \Omega$ & {$\left[6.8 \times 10^{-10}\right]$} \\
\hline
\end{tabular}

\section{The $h$ group}

The data group related to elementary charge $e$ and the Planck constant $h$ is formed by the fact that we know with a high accuracy data in Table 2. Since the knowledge of $\alpha, h N_{A}, h / m_{e}$ sets certain constraints on the results on $e, h, N_{A}, m_{e}$ and their various combinations $\left(e / h, e / m_{e}\right.$, $F=e N_{A}, \mu_{B}=e \hbar /\left(2 m_{e}\right)$ etc. $)$, the determination of any of them produces an input for all of them. The related recommended values [1] are summarized in Table 3. 
Table 3. Some $h$ related data [1]. Here, $u_{r}$ stands for the fractional uncertainty.

\begin{tabular}{lccc}
\hline Quantity & Symbol & Value & $u_{r}$ \\
\hline Planck constant & $h$ & $6.62606896(33) \times 10^{-34} \mathrm{~J} \mathrm{~s}$ & {$\left[5.0 \times 10^{-8}\right]$} \\
elementary charge & $e$ & $1.602176487(40) \times 10^{-19} \mathrm{C}$ & {$\left[2.5 \times 10^{-8}\right]$} \\
Avogadro constant & $N_{A}$ & $6.02214179(30) \times 10^{23} \mathrm{~mol}^{-1}$ & {$\left[5.0 \times 10^{-8}\right]$} \\
Faraday constant & $F=e \cdot N_{A}$ & $96485.3399(24) \mathrm{C} \mathrm{mol}^{-1}$ & {$\left[2.5 \times 10^{-8}\right]$} \\
Josephson constant & $K_{\mathrm{J}}$ & $483597.891(12) \times 10^{9} \mathrm{~Hz} \mathrm{~V}^{-1}$ & {$\left[2.5 \times 10^{-8}\right]$} \\
electron charge to mass quotient & $e / m_{e}$ & $1.758820150(44) \times 10^{11} \mathrm{C} \mathrm{kg}^{-1}$ & {$\left[2.5 \times 10^{-8}\right]$} \\
electron mass & $m_{e}$ & $9.10938215(45) \times 10^{-31} \mathrm{~kg}$ & {$\left[5.0 \times 10^{-8}\right]$} \\
Bohr magneton & $\mu_{B}$ & $927.400915(23) \times 10^{-26} \mathrm{~J} \mathrm{~T}^{-1}$ & {$\left[2.5 \times 10^{-8}\right]$} \\
\hline
\end{tabular}

The crucial input data are presented in Fig. 2. They include ten data points, achieved by five independent methods. Most of them are due to electric measurements, except an X-ray crystal density (XRCD) study of a Si crystal.

The most important development in the field was due to progress with the NIST watt balance [15]. This result is in many details uncorrelated with the older NIST measurement and
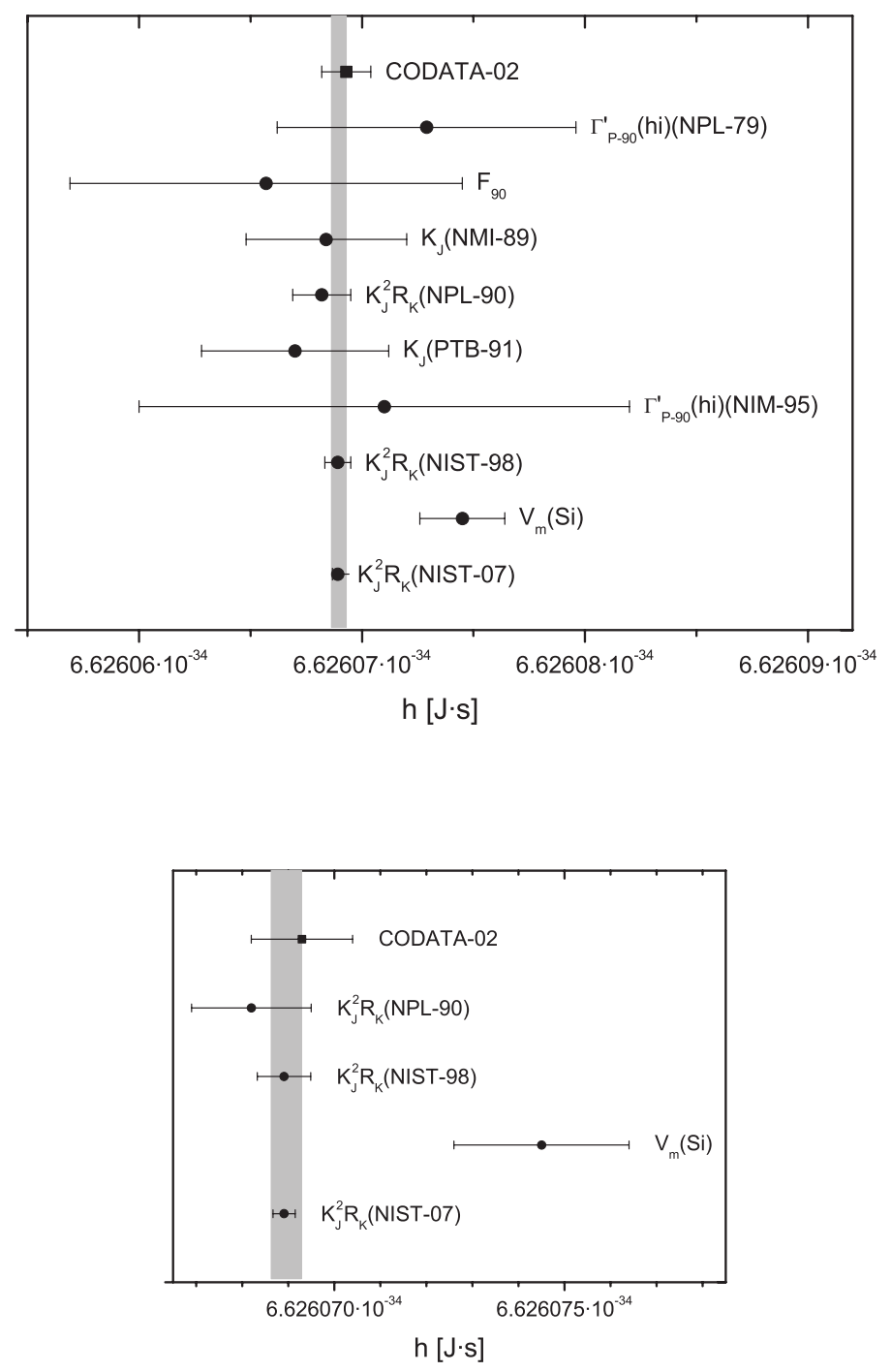

Fig. 2. Determination of the Planck constant $h$ in the CODATA-2006 adjustment [1]. The labels are similar to those in [1]. The vertical band stands for the adjusted CODATA-2006 value. The most accurate data from the watt balances and XRCD measurement are presented in the bottom plot with a magnified scale. 
has a higher accuracy. It dominates in the determination of $h$. However, the electric determinations of the Planck constant strongly disagree with XRCD determination of $N_{A}$. Because of this discrepancy in the evaluation of the data an extended-uncertainty method is applied. The problem of consistency of the world data remains unresolved.

\section{Independent constants}

There is a number of the fundamental constants which do not affect the adjusted values of the others or their effect is marginal. Most important of them are collected in Table 4.

Table 4. 'Independent' constants which are marginally involved into the adjustment procedure [1].

\begin{tabular}{lccc}
\hline Quantity & Symbol & Value & $u_{r}$ \\
\hline Newtonian constant of gravitation & $G$ & $6.67428(67) \times 10^{-11} \mathrm{~m}^{3} \mathrm{~s}^{-2} \mathrm{~kg}^{-1}$ & {$\left[1.0 \times 10^{-4}\right]$} \\
molar gas constant & $R$ & $8.314472(15) \mathrm{J} \mathrm{K}^{-1} \mathrm{~mol}^{-1}$ & {$\left[1.7 \times 10^{-6}\right]$} \\
Boltzmann constant & $k$ & $1.3806504(24) \times 10^{-23} \mathrm{~J} \mathrm{~K}^{-1}$ & {$\left[1.7 \times 10^{-6}\right]$} \\
\hline
\end{tabular}

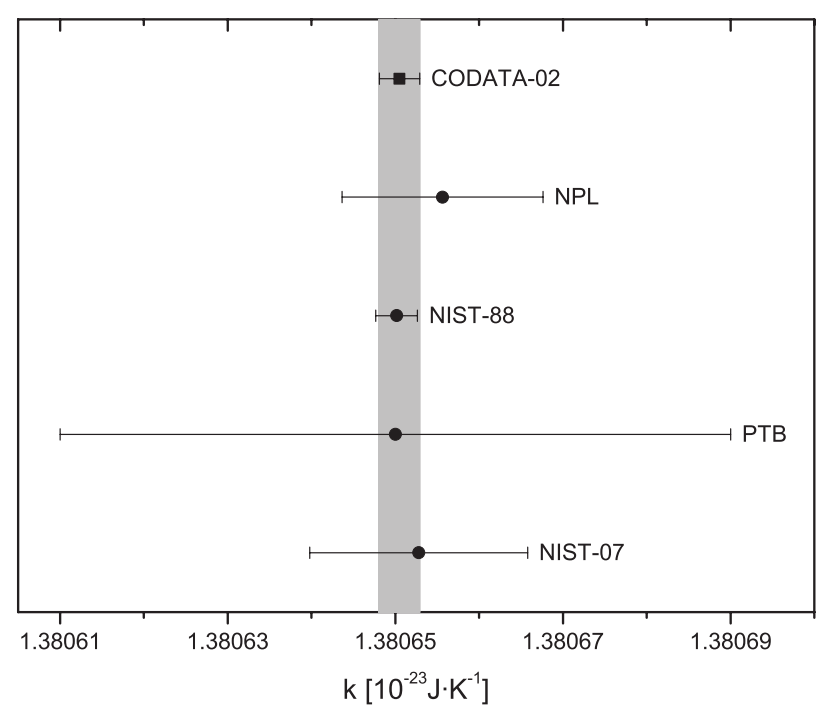

Fig. 3. Determination of the Boltzmann constant $k$ in the CODATA-2006 adjustment [1]. The labels are similar to those in [1]. The vertical band stands for the adjusted CODATA-2006 value.

The status of the Boltzmann constant $k$ and the molar gas constant $R$ did not really change during nearly two last decades. The present results for $k$ are collected in Fig. 3 . The only progress is recent appearance of two preliminary results (from PTB and NIST) and we hope that their accuracy will improve in future.

The changes with the Newtonian constant of gravitation, $G$, during the last decade is rather dramatic. Despite of its great fundamentality (that is, perhaps, one of three most fundamental constants we experimentally deal with; the others are indeed $c$ and $h$ ), it is marginally involved in precision measurements.

Most accurate tests of general relativity (GR) deal with a product of $G$ and a certain 'big mass' (of Sun, Earth) etc. The product is often known very accurately, but it is not fundamental. $G$ is fundamental, but it can be measured only in dedicated experiments, where accuracy is much lower than can be designed for the GR tests. 
In Fig. 4 we present results of the four last adjustments (note, that the CODATA-1986 value [3] is included into the summary plot for CODATA-1998 [4]).
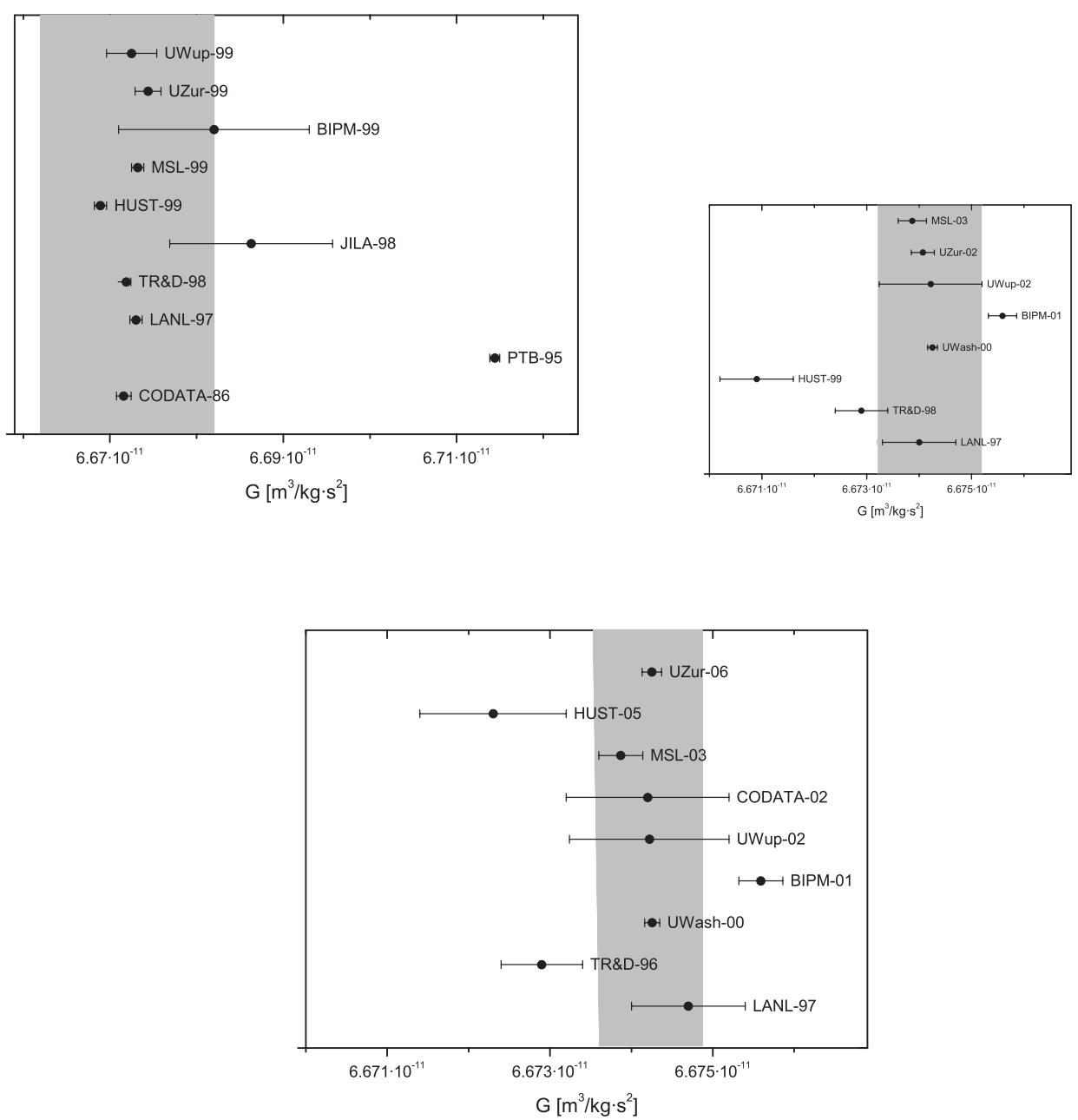

Fig. 4. The gravitation constant $G$ in the CODATA-1998 [4] (left), CODATA-2002 [5] (right) CODATA-2006 [1] (bottom) adjustments. The labels follow the quoted papers. The vertical bands stand for the contemporary adjusted CODATA values.

The accuracy of the adjustment is not determined by the uncertainty of particular data points, but by their scatter. To establish a reasonable agreement between different points we have to expand the uncertainty. In 1998 the CODATA uncertainty was increased because of the data scatter and specifically because of the PTB-95 measurement. In the 2002 evaluation more data confirming the central cluster appeared and after consulting with PTB it was agreed to remove the PTB-95 result from the adjustment. The data of 2006 are different with data of 2002 basically because of finalizing the data analysis of measurements, preliminary data of which were evaluated in the adjustment-2002.

\section{Electric data}

Due to practical applications it is important to consider 'electric' data in more details. Such data are widely used in the determination of $\alpha$ (Fig. 1) and $R_{\mathrm{K}}$ and of $h$ (Fig. 2) and $K_{\mathrm{J}}$. This 
opportunity is open because in electric measurements one uses 'practical' units, i.e. units in which numerical values of the von Klitzing constant $R_{\mathrm{K}}$ and the Josephson constant $K_{\mathrm{J}}$ are known exactly by definition [16].

Use of practical units means that all voltages, resistances and currents were measured against electric standards based on the quantum Hall effect and Josephson effect. The present status in the determination of $R_{\mathrm{K}}$ and $K_{\mathrm{J}}$, which is very similar to status for $\alpha$ and $h$, respectively, is summarized in Fig. 5.
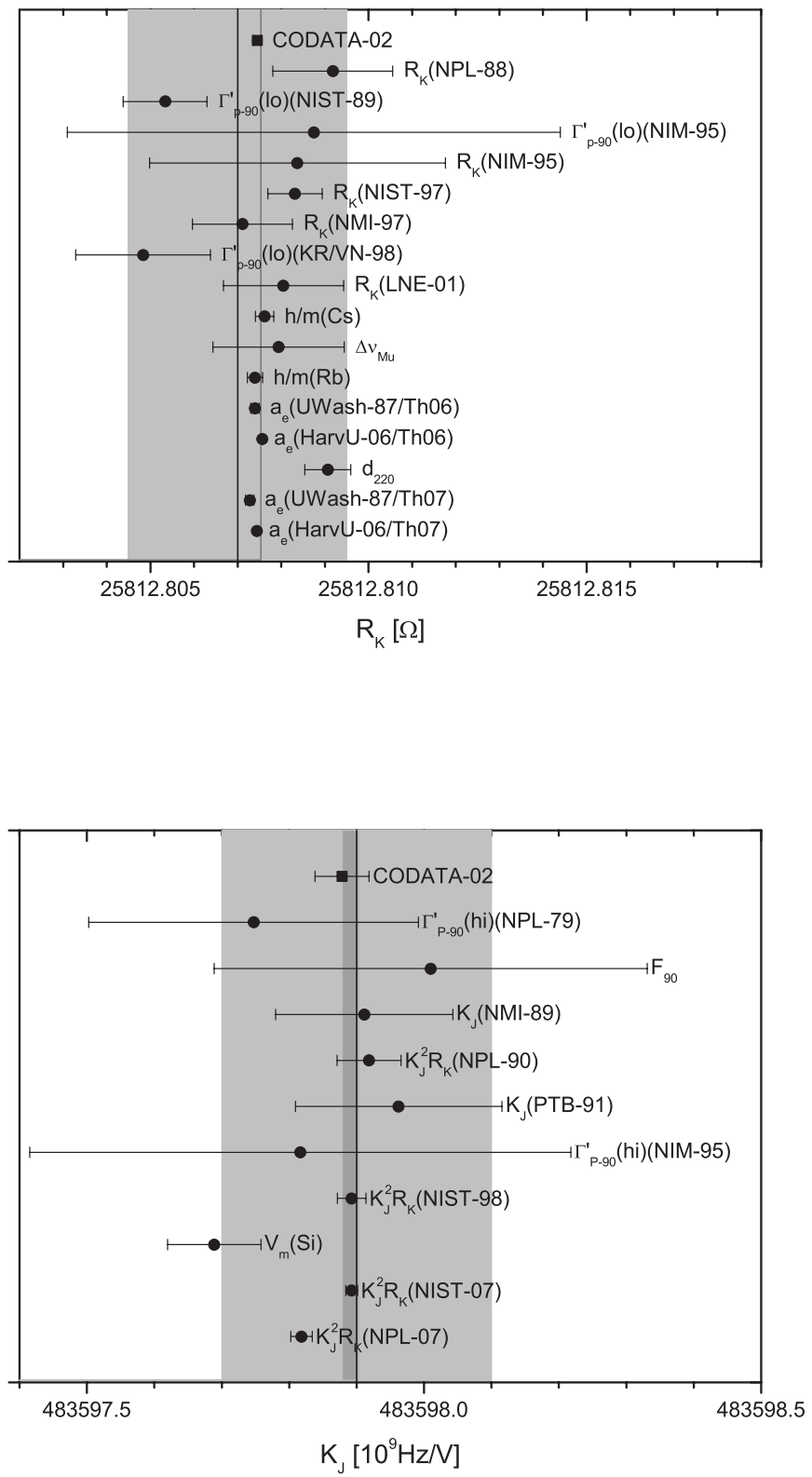

Fig. 5. Determination of the von Klitzing constant $R_{\mathrm{K}}$ and the Josephson constant $K_{\mathrm{J}}$ in the CODATA-2006 adjustment [1] and afterwards (see points labeled as $a_{e}(\ldots /$ Th07) $[17,18]$ and $\left.K_{\mathrm{J}}^{2} R_{\mathrm{K}}(\mathrm{NPL}-07)[19]\right)$. The labels are similar to those in [1]. The narrow vertical bands stand for the adjusted CODATA-2006 values. The broad vertical bands indicate the CIPM recommended value [16] of $R_{\mathrm{K}}$ and $K_{\mathrm{J}}$ with a line for the central value suggesting $\Omega_{\mathrm{SI}}=\Omega_{90}$ and $\mathrm{V}_{\mathrm{SI}}=\mathrm{V}_{90}$. 
Because of great importance of two mentioned quantum effects in metrology and because of uncertainty in the question how accurate are relations between $e$ and $h$ and $R_{\mathrm{K}}$ and $K_{\mathrm{J}}$, in two most recent adjustments $[1,5]$ an additional test was performed. It was suggested to use instead of simple relations (1), more complicated equations

$$
\begin{aligned}
& R_{\mathrm{K}}=\left(1+\epsilon_{\mathrm{K}}\right) \times \frac{h}{e^{2}}=\left(1+\epsilon_{\mathrm{K}}\right) \times \frac{\mu_{0} c}{2 \alpha} \\
& K_{\mathrm{J}}=\left(1+\epsilon_{\mathrm{J}}\right) \times \frac{2 e}{h}=\left(1+\epsilon_{\mathrm{J}}\right) \times\left(\frac{8 \alpha}{\mu_{0} c h}\right)^{1 / 2},
\end{aligned}
$$

with $\epsilon_{\mathrm{K}}$ and $\epsilon_{\mathrm{J}}$ as adjustable parameters.

The result of such an evaluation gave a certain range of possible $\epsilon$ parameters more or less consistent with zero. The procedure is not straightforward because $\epsilon_{\mathrm{J}}$ data, which have marginal importance for the conventional procedure because of their statistical weight or because of their questionable status, become important here.

Shortly speaking, for $\epsilon_{\mathrm{K}}$ we can look into the top plot in Fig. 5. A systematic discrepancy between non-electric data (marked as $a_{e}$ or $h / m$ ) with data from calculable-capacitor measurements (marked by $R_{\mathrm{K}}$; see [20] on details) would mean a non-zero $\epsilon_{\mathrm{K}}$. The result of [1] is

$$
\epsilon_{\mathrm{K}} \simeq 2(2) \times 10^{-8}
$$

which marginally depends, on which else data are included.

The situation with $\epsilon_{\mathrm{J}}$ is not that simple. The top plot in Fig. 5 would indicate a non-zero $\epsilon_{\mathrm{J}}$, if the gyromagnetic measurement at low magnetic field (marked as $\Gamma$ (lo)) would systematically disagree with results of two methods mentioned due to $\epsilon_{\mathrm{K}}$. In the bottom plot, if $\epsilon_{\mathrm{J}} \neq 0$, we should discover, that the results from watt balances (marked as $K_{\mathrm{J}}^{2} R_{\mathrm{K}}$; see [21] on details) would disagree with the XRCD data point (marked as $V_{m}(\mathrm{Si})$ ). In both cases there are non-zero signals $\left(\epsilon_{\mathrm{J}}(\Gamma)=-2.8(1.0) \times 10^{-7}\right.$ and $\left.\epsilon_{\mathrm{J}}\left(V_{m}\right)=+4.1(1.4) \times 10^{-7}\right)$, but they are not consistent. We should choose which to follow. As a result, our conservative estimation based on [1] is

$$
-3 \times 10^{-7} \leq \epsilon_{\mathrm{J}} \leq 5 \times 10^{-7} .
$$

Due to importance of these constraints we have to properly interpret them. Both values, $R_{\mathrm{K}}$ and $K_{\mathrm{J}}$, are electric properties of matter, which can in principle have two kinds of corrections, namely universal material-independent and peculiar material-dependent corrections. The constraints from [1] are applicable to the material-independent effect. We note that a QED series for fundamental values are similar to that for the anomalous magnetic moment of electron, which is of the form ${ }^{2}$

$$
a_{e}=C_{1}\left(\frac{\alpha}{\pi}\right)+C_{2}\left(\frac{\alpha}{\pi}\right)^{2}+C_{3}\left(\frac{\alpha}{\pi}\right)^{3}+\ldots
$$

which coefficients are of the order of unity and there is a small parameter $\alpha / \pi \simeq 2.3 \times 10^{-3}$. If we suggest such a correction for $R_{\mathrm{K}}$ and $K_{\mathrm{J}}$, then, based on the numerical order of magnitude of possible correction, we interpret the constraint (5) and (6) as

$$
C_{1}\left(R_{\mathrm{K}}\right)=0, \quad C_{2}\left(R_{\mathrm{K}}\right)=0, \quad C_{3}\left(R_{\mathrm{K}}\right) \simeq 2(2) ;
$$

and

$$
C_{1}\left(K_{\mathrm{J}}\right)=0, \quad C_{2}\left(K_{\mathrm{J}}\right)=0, \quad-24 \leq C_{3}\left(K_{\mathrm{J}}\right) \leq 40
$$

and there is no way to expect reasonable non-zero values of $C_{1,2}$. Meanwhile, that is very unlikely to expect that the series would start with $C_{3}$ as the first non-zero coefficient.

\footnotetext{
${ }^{2}$ One may have in mind a very different structure of a series for hydrogenic transitions. Those series are in a sense an example of material-dependent corrections, because $\alpha$ appears there not only as a QED loop parameter (cf. (7)) as the strength of the Coulomb potential and the numerical coefficients depend on a transition and atom under consideration.
} 
That means that it is very likely that the evaluation in [1] ruled out any reasonable opportunity for material-independent corrections.

A study of the material-dependent effect can be done in a different matter and in fact many tests of this kind have been performed (see, e.g., [22,23]). The related limits on violation of naive relations (1) are at the level essentially below than one part in $10^{10}$ which is sufficient for any practical applications.

Nevertheless, we have to stress that such tests should be continued. There is no just 'material-dependent' corrections. The correction should depend on parameters of materials. It may easily happen that two different materials possess the same values of some parameters or studied under the same conditions (e.g., at the same temperature). To really rule out any material-dependent correction at the level of one part in $10^{10}$ we should be sure that all parameters under question vary in a broad range. The contemporary compilations of a study of universality of $R_{\mathrm{K}}$ and $K_{\mathrm{J}}$ do not present enough data for such conclusions. We hope such analysis of existing data will be done and either strong constraint will be derived or it will be concluded which experiments are necessary for such constraints.

\section{Progress in determination of fundamental constants}

Concluding a review of the CODATA-2006 recommended data we present some comparison of the most recent data with the earlier recommended sets.

The progress is summarized in Fig. 6 and Table 5, where progress of most constants discussed in previous sections is presented.

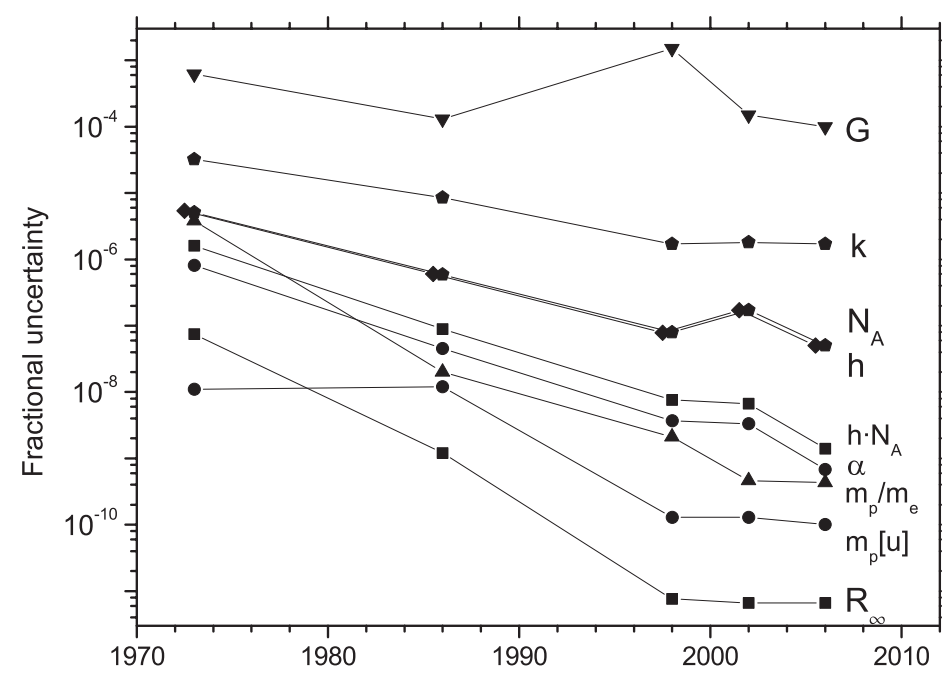

Fig. 6. Progress in determination of fundamental constants.

One can note that the accuracy not necessarily increases with time (see Fig. 6). That happens either because of discovery of additional sources of the uncertainty or because of appearance of new data which are not consistent with the previous results. Appearance of new data and new corrections may also shift some values so much that their difference is above their uncertainty.

Because of that one may ask what the main result of the adjustments is and how to utilize the recommended values.

\section{Legacy of the CODATA adjustments}

The most important part of the adjustment is neither a compilation of the world data nor their evaluation with the least square method. The heart of the adjustment is the critical examination of the data. 
Table 5. Progress in determinatiton of the most important fundamental constants in 1998-2006 [1,4,5].

\begin{tabular}{lccc}
\hline Quantity & \multicolumn{3}{c}{ Recommended values } \\
[unit] & $1998[4]$ & $2002[5]$ & $2006[1]$ \\
\hline$R_{\infty}\left[\mathrm{m}^{-1}\right]$ & $10973731.568549(83)$ & $10973731.568525(73)$ & $10973731.568527(73)$ \\
$m_{p}[\mathrm{u}]$ & $1.00727646688(13)$ & $1.00727646688(13)$ & $1.00727646677(10)$ \\
$m_{p} / m_{e}$ & $1836.1526675(39)$ & $1836.15267261(85)$ & $1836.15267247(80)$ \\
$\alpha^{-1}$ & $137.03599976(50)$ & $137.03599911(46)$ & $137.03599968(9)$ \\
$h N_{A}\left[\mathrm{~J} \mathrm{~s} \mathrm{~mol}^{-1}\right]$ & $3.990312689(30) \times 10^{-10}$ & $3.990312716(27) \times 10^{-10}$ & $3.9903126821(57) \times 10^{-10}$ \\
$h[\mathrm{~J} \mathrm{~s}]$ & $6.62606876(52) \times 10^{-34}$ & $6.6260693(11) \times 10^{-34}$ & $6.62606896(33) \times 10^{-34}$ \\
$N_{A}\left[\mathrm{~mol}^{-1}\right]$ & $6.02214199(47) \times 10^{23}$ & $6.0221415(10) \times 10^{23}$ & $6.02214179(30) \times 10^{23}$ \\
$e[\mathrm{C}]$ & $1.602176462(63) \times 10^{-19}$ & $1.60217653(14) \times 10^{-19}$ & $1.602176487(40) \times 10^{-19}$ \\
$k\left[\mathrm{~J} \mathrm{~K}^{-1}\right]$ & $1.3806503(24) \times 10^{-23}$ & $1.3806505(24) \times 10^{-23}$ & $1.3806504(24) \times 10^{-23}$ \\
$G\left[\mathrm{~m}^{3} \mathrm{~s}^{-2} \mathrm{~kg}^{-1}\right]$ & $6.673(10) \times 10^{-11}$ & $6.6742(10) \times 10^{-11}$ & $6.67428(67) \times 10^{-11}$ \\
\hline
\end{tabular}

This examination helps to learn whether the world data are trustworthy and whether they are consistent. Part of the measurements involve various standards (mainly, they deal with electric standards) and their tests is of top priority for the metrological community.

Thus, the adjustment is first of all an overall cross-check of the existing data. In contrast to many other compilations of the reference data, the CODATA papers [1-5] present all important details of this examination.

Actually, that answers the question how to use the data. If one really needs an accurate value, we would not recommend to apply the CODATA values. Instead, we recommend to use the data examination in the most recent CODATA adjustment. For instance, if a certain calculation is very sensitive to the accurate value of $\alpha$ and it is needed to compare it with an experimental result, we recommend to use the analysis (see Fig. 1) and calculate a new value of $\alpha$ from the mentioned theory and experiment.

Meanwhile, the recommended values are the best choice if accurate values are not really necessary, but they are very helpful to avoid a scatter in results.

A similar approach is demonstrated, e.g., by CIPM when it is necessary to produce CIPM recommended values of $R_{\mathrm{K}}$ and $K_{\mathrm{J}}$. They use the CODATA data and their analysis, but for pragmatic reasons they choose a much more conservative uncertainty estimation.

For more discussions on the question see $[8,9,24]$.

Completing the paper, we would like to raise two issues.

Firstly, we note that the CODATA task group on fundamental constants is not a team of magicians. The group performs the best possible analysis of the existing world data and the best possible evaluation. However, not all problems inside the measurements and calculations can be understood 'from outside'. An example of this is a discovery of certain corrections in the theory of the anomalous magnetic moment of electron [17], which should shift the related value of the fine structure constants by approximately five standard deviations [18]. Ironically, the result was published three months after the CODATA-2006 values were published on-line [1], but before the paper [1] was even submitted.

The other question to consider is the 'whole picture'. When we do physics, we apply various approximations and some models. In a sense, the whole physical description of Nature is kind of a 'big approximation'. It might happen that different approximations are not consistent. We all know that about a century ago a successful mechanical theory was not consistent with another successful theory, which described electromagnetic effects. And we remember the consequence.

Therefore, it is an important problem to check the overall consistency and the CODATA adjustment allows to verify whether we have certain inconsistency at present. Likely, that is the most accurate test which touches a quite broad range of phenomena.

The result of this test [1] does not show any significant discrepancy. The particular results obtained by different methods are essentially consistent.

The author is a member of the CODATA task group on fundamental physical constants and is grateful to his colleagues for fruitful discussions. The data are officially recommended by the group and published 
in [1]; the interpretation of certain details does not necessary coincide with the group opinion and presents a personal author's view.

The author is grateful to the organizers of the International School "Quantum Metrology and Fundamental Constants" at École de physique in Les Houches for warm atmosphere and fruitful discussions there. The work was supported in part by RFBR under grant \\# 08-02-13516-ofi-z.

\section{References}

1. P.J. Mohr, B.N. Taylor, D.B. Newell, Rev. Mod. Phys. 80, 633 (2008); see also P.J. Mohr, B.N. Taylor, D.B. Newell, Phys. Today 60, 52 (2007) and on-line at http://physics.nist.gov/cuu/Constants/index.html

2. E.R. Cohen, B.N. Taylor, J. Phys. Chem. Rev. Data 2, 663 (1973)

3. E.R. Cohen, B.N. Taylor, Rev. Mod. Phys. 59, 1121 (1987)

4. P.J. Mohr, B.N. Taylor, Rev. Mod. Phys. 72, 351 (2000)

5. P.J. Mohr, B.N. Taylor, Rev. Mod. Phys. 77, 1 (2005)

6. See for the site: http://www. codata.org/taskgroups/TGfundconst/index.html

7. P.J. Mohr, B.N. Taylor, Phys. Today (August 2000) BG6; (March 2001), p. 29

8. S.G. Karshenboim, Physics-Uspekhi 48, 255 (2005)

9. S.G. Karshenboim, in Precision Physics of Simple Atoms and Molecules, edited by S.G. Karshenboim (Springer, Berlin, Heidelberg, 2007), p. 35

10. The International System of Units (SI), BIPM, Sèvres, 2006; also available on-line at http://www.bipm.org

11. T. Kinoshita, M. Nio, Phys. Rev. D 73, 013003 (2006)

12. B. Odom, D. Hanneke, B. D'Urso, G. Gabrielse, Phys. Rev. Lett. 97, 030801 (2006)

13. G. Gabrielse, D. Hanneke, T. Kinoshita, M. Nio, B. Odom, Phys. Rev. Lett. 99, 039902 (2006)

14. P. Cladé, E. de Mirandes, M. Cadoret, S. Guellati-Khélifa, C. Schwob, F. Nez, L. Julien, F. Biraben, Phys. Rev. Lett. 96, 033001 (2006)

15. R.L. Steiner, E.R. Williams, D.B. Newell, R. Liu, Metrologia 42, 431 (2005); R.L. Steiner, E.R. Williams, R. Liu, D.B. Newell, IEEE Trans. IM-56, 592 (2007)

16. T.J. Quinn, Metrologia 26, 70 (1989); T.J. Quinn, Metrologia 38, 89 (2001); T.J. Quinn, Metrologia 26, 69 (1989)

17. T. Aoyama, M. Hayakawa, T. Kinoshita, M. Nio, Phys. Rev. Lett. 99, 110406 (2007)

18. G. Gabrielse, D. Hanneke, T. Kinoshita, M. Nio, B. Odom, Phys. Rev. Lett. 99, 039902 (2007)

19. I.A. Robinson, B.P. Kibble, Metrologia 44, 427 (2007)

20. H. Bachmair, Eur. Phys. J. Special Topics 172, 257 (2009)

21. A. Eichenberger, G. Genevès, P. Gournay, Eur. Phys. J. Special Topics 172, 363 (2009)

22. F. Delahaye, D. Dominguez, F. Alexandre, J.P. Andre, J.P. Hirtz, M. Razeghi, Metrologia 22, 103 (1986); A. Hartland, K. Jones, J.M. Williams, B.L. Gallagher, T. Galloway, Phys. Rev. Lett. 66, 969 (1991); B. Jeanneret, B. Jeckelmann, H.-J. Bühlmann, R. Houdré, M. Ilegems, IEEE Trans. IM-44, 254 (1995); B. Jeckelmann, D. Inglis, B. Jeanneret, IEEE Trans. IM-44, 269 (1995); B. Jeckelmann, B. Jeanneret, D. Inglis, Phys. Rev. B 55, 13124 (1997); B. Jeckelmann, et al., IEEE Trans. IM-50, 219 (2001); F. Schopfer, W. Poirier, J. Appl. Phys. 102, 54903 (2007); see also contribution by B. Jeanneret, S.P. Benz, Eur. Phys. J. Special Topics 172, 181 (2009)

23. J.S. Tsai, A.K. Jain, J.E. Lukens, Phys. Rev. Lett. 51, 316 (1983); J. Neimeyer, L. Grimm, C.A. Hamilton, R.L. Steiner, IEEE Electron Device Lett. 7, 44 (1986); R.L. Kautz, F.L. Lloyd, Appl. Phys. Lett. 51, 2043 (1987); A.K. Jain, J.E. Lukens, J.S. Tsai, Phys. Rev. Lett. 58, 1165 (1987); I.Y. Krasnopolin, R. Behr, J. Neimeyer, Supercond. Sci. Technol. 15, 1034 (2001); see also contribution by W. Poirier, F. Schopfer, Eur. Phys. J. Special Topics 172, 207 (2009)

24. S.G. Karshenboim, Can. J. Phys. 83, 767 (2005) 